

К. Морози, Д. Тондо, Разделение переменных в мультигамильтоновых системах. Применение к лагранжеву волчку, ТМФ, 2003, том 137, номер 2, 226-238

DOI: https://doi.org/10.4213/tmf268

Использование Общероссийского математического портала Math-Net.Ru подразумевает, что вы прочитали и согласны с пользовательским соглашением

http://www . mathnet.ru/rus/agreement

Параметры загрузки:

IP : 52.6 .47 .48

26 апреля 2023 г., 11:53:59 
ТЕОРЕТИЧЕСКАЯ

И МАТЕМАТИЧЕСКАЯ

ФИЗИКА

Том 137, № 2

ноябрь, 2003

(C) 2003 г.

К. Морози*, Дж. Тондо ${ }^{\dagger}$

\section{РАЗДЕЛЕНИЕ ПЕРЕМЕННЫХ В МУЛЬТИГАМИЛЬТОНОВЫХ СИСТЕМАХ. ПРИМЕНЕНИЕ К ЛАГРАНЖЕВУ ВОЛЧКУ}

Отталкиваясь от трехгамильтоновой формулировки лагранжева волчка в шестимерном фазовом пространстве, удается построить редукцию векторного поля и пуассоновых тензоров. Явно показано, что после редукции на каждый из симплектических листов векторное поле лагранжева волчка допускает разделение переменных в смысле Гамильтона-Якоби.

Ключевые слова: лагранжев волчок, гамильтонова формулировка, разделяемость переменHых.

\section{1. ВВЕДЕНИЕ}

В данной статье завершается исследование лагранжева волчка (ЛВ) как квазибигамильтоновой (КБГ) системы, начатое в работе [1] (содержащей детальное изложение вопроса). В работе [1] было показано, что трехгамильтонова структура ЛВ, задаваемая на шестимерном фазовом пространстве тремя согласованными пуассоновыми тензорами $P_{0}, P_{1}$ и $P_{2}$, может быть редуцирована на четырехмерное фазовое пространство. При попытке исключить функции Казимира пуассоновых тензоров, фиксируя их значения, мы сталкиваемся с типичной ситуацией, которая также возникает и в других конечномерных интегрируемых системах [2]: на каждњй из симплектических листов $S_{0}$, $S_{1}$ и $S_{2}$ можно ограничить только лишь векторное поле $X_{L}$ и соответствующий этому полю пуссонов тензор, но нельзя ограничить полную трехгамильтонову структуру, которая нарушается при таком ограничении. Тем не менее векторное поле ЛВ $X_{L}$, будучи ограниченным на симплектический лист $S_{0}$ пуассонова тензора $P_{0}$, допускает формулировку в виде КБГ-системы, что приводит к разделимости переменных в этой структуре в смысле Гамильтона-Якоби (ГЯ).

В данной статье будет показано, что это свойство векторного поля $X_{L}$ оказывается более общим. На самом деле свойство КБГ возникает и в том случае, когда ограниче-

\footnotetext{
* Dipartimento di Matematica, Politecnico di Milano, P.za L. da Vinci 32, I-20133 Milan, Italy. E-mail: carmor@mate.polimi.it

${ }^{\dagger}$ Dipartimento di Scienze Matematiche, Università di Trieste, via A. Valerio 12/1, I-34127 Trieste, Italy. E-mail: tondo@univ.trieste.it
} 
ние производится на любой симплектический лист второго пуассонова тензора $P_{1}$; более того, исследуя некоторые свойства КБГ-модели, можно явно построить переменные разделения для ограничений структуры $X_{L}$ на любой симплектический лист третьего пуассонова тензора $P_{2}$. Интересно отметить, что в обоих случаях переменные разделения получаются с использованием несимплектических отображений.

Статья имеет следуюшую структуру. В разделах 2 и 3 обсуждаются некоторые свойства общей трехгамильтоновой структуры с деформациями и свойства КБГ-модели с точки зрения приложения к ЛВ. В разделах 4 и 5 содержится краткий обзор трехгамильтоновой структуры для ЛВ, деформационного поля и их редукций. Наконец, в разделе 6 с использованием результатов раздела 3 показано, что поле $X_{L}$ может быть представлено в форме, допускаюшей разделение переменных, если это поле ограничено на любой симплектический лист одного из трех пуассоновых тензоров.

\section{2. ДЕФОРМАЦИИ МУЛЬТИГАМИЛЬТОНОВЫХ СТРУКТУР И НАСЛЕДСТВЕННЫЕ ОПЕРАТОРЫ}

Сделаем следующие предположения:

1. Многообразие $\left(M, P_{2}, \tau\right)$ представляет собой пуассоново многообразие с деформацией, т.е. $M$ есть дифференциальное многообразие с пуассоновым тензором $P_{2}$ и векторным полем $\tau$ таким, что производная Ли $P_{1}=L_{\tau}\left(P_{2}\right)$ тензора $P_{2}$ относительно поля $\tau$ сама оказывается пуассоновым тензором. Из этого предположения следует, что комбинация $P_{2}-\lambda P_{1}$ есть пуассонов пучок.

2. Пуассонов тензор $P_{1}$ точен относительно поля $\tau$, т.е. $L_{\tau}^{2}\left(P_{1}\right)=0$. Отсюда следует, что производная Ли $P_{0}=L_{\tau}\left(P_{1}\right)=L_{\tau}^{2}\left(P_{2}\right)$ сама оказывается пуассоновым тензором, совместным как с $P_{1}$, так и с $P_{2}$.

Из этих предположений следует

Лемма 2.1. Многообразие $\left(M, P_{0}, P_{1}, P_{2}\right)$ представляет собой трехгамильтоново многообразие, т.е. линейная комбинация $P_{0}-\lambda P_{1}-\mu P_{2}$ сама представляет собой пуассонов тензор при всех значениях постоянных коэффициентов $\lambda u \mu$.

ЗАмЕЧАНИЕ. Как легко проверить, если существует трехгамильтонова структура $\left(\widetilde{P}_{0}, \widetilde{P}_{1}, \widetilde{P}_{2}\right)$ такая, что $L_{\tau}\left(\widetilde{P}_{0}\right)=0, L_{\tau}\left(\widetilde{P}_{1}\right)=\alpha \widetilde{P}_{0}$ и $L_{\tau}\left(\widetilde{P}_{2}\right)=\beta \widetilde{P}_{1}+\gamma \widetilde{P}_{0}$ с постоянными коэффициентами $\alpha, \beta$ и $\gamma$, то при каждом значении параметра $a$ величины $P_{0}=\widetilde{P}_{0}, P_{1}=$ $(1 / \alpha) \widetilde{P}_{1}+(\gamma / \alpha \beta) \widetilde{P}_{0}$ и $P_{2}=(a / \alpha \beta) \widetilde{P}_{2}$ удовлетворяют соотношениям деформации

$$
L_{\tau}\left(P_{2}\right)=a P_{1}, \quad L_{\tau}\left(P_{1}\right)=P_{0}, \quad L_{\tau}\left(P_{0}\right)=0
$$

Утверждение следуюшей леммы задает достаточное условие для сушествования проекции трехгамильтоновой структуры при погружении.

Лемма 2.2. Пусть отображение $\pi: M \mapsto M^{\prime}$ задает сюрвективное погружение на многообразие $M^{\prime}$. Если обе структуры $P_{2}$ и $\tau$ имеют хорошо опеределенние проекиии на $M^{\prime}$, то и полная трехгамильтонова структура сохраняется при погружсении $\pi$. Обозначим через $P_{0}^{\prime}, P_{1}^{\prime}$ и $P_{2}^{\prime}$ редуцированные тензоры и через $\tau^{\prime}$ 
редуцированное векторное поле. Тогда получим

$$
L_{\tau^{\prime}}\left(P_{2}^{\prime}\right)=a P_{1}^{\prime}, \quad L_{\tau^{\prime}}\left(P_{1}^{\prime}\right)=P_{0}^{\prime}, \quad L_{\tau^{\prime}}\left(P_{0}^{\prime}\right)=0
$$

Как известно, в случае, когда редуцированный пуассонов тензор $P_{0}^{\prime}$ имеет нулевое ядро (обратный к нему тензор при этом симплектичен), то на многообразии $M^{\prime}$ оператор $N=P_{1}^{\prime} P_{0}^{\prime-1}$ представляет собой наследственный оператор, т.е. его кручение Нийенхейса [3] оказывается равным нулю. Наша задача теперь состоит в том, чтобы найти такие ограничения на деформацию $\tau$, которые бы приводили к тому, чтобы оператор $N$ действовал как оператор рекурсии (в направлении, противоположном направлению производных Ли относительно поля $\tau$ ) для редуцированной трехгамильтоновой структуры на многообразии $M^{\prime}$, а также отображал $P_{1}^{\prime}$ в $P_{2}^{\prime}$ (возможно, с точностью до постоянного множителя): $N P_{1}^{\prime}=\lambda P_{2}^{\prime}$ при постоянной $\lambda$. Для этого можно использовать следуюший результат.

Лемма 2.3. Пусть задань векторное поле $\tau$ на многообразии $M$ и его редукиия $\tau^{\prime}$ на $M^{\prime}$. Рассмотрим уравнение $L_{\tau^{\prime}}\left(Q^{\prime}\right)=0$ и будем искать такое его решение $Q^{\prime}$, которое бълло бъ кососимметричныцм $(2,0)$-тензором. Если $P_{0}^{\prime}$ представляет собой общее рещение ( с точностью до мультипликативной константы), т.е. $L_{\tau^{\prime}}\left(Q^{\prime}\right)=0 \Rightarrow Q^{\prime}=\alpha P_{0}^{\prime}$, то тензор $N:=P_{1}^{\prime} P_{0}^{\prime-1}$, заданный на многообразии $M^{\prime}$, обладает свойством

$$
N P_{1}^{\prime}=\frac{2}{a} P_{2}^{\prime}+\beta P_{0}^{\prime}, \quad \beta=\text { const } .
$$

ДоКАЗАТЕЛЬСТво. Согласно условиям (2.2) и предыдушим предположениям уравнения $L_{\tau^{\prime}}\left(Q^{\prime}\right)=P_{0}^{\prime}$ и $L_{\tau^{\prime}}\left(Q^{\prime}\right)=P_{1}^{\prime}$ обладают соответственно решениями $Q^{\prime}=P_{1}^{\prime}+\alpha P_{0}^{\prime}$ и $Q^{\prime}=(1 / a) P_{2}^{\prime}+\alpha P_{0}^{\prime}$. Вследствие равенства $L_{\tau^{\prime}}(N)=I$ получим

$$
\frac{1}{2-a} L_{\tau^{\prime}}\left(N P_{1}^{\prime}-P_{2}^{\prime}\right)=P_{1}^{\prime} \Rightarrow \frac{1}{2-a}\left(N P_{1}^{\prime}-P_{2}^{\prime}\right)=\frac{1}{a} P_{2}^{\prime}+\alpha P_{0}^{\prime},
$$

откуда следует утверждение (2.3) с параметром $\beta=(2-a) \alpha$.

Лемма 2.4. Пусть задана трехгамильтонова структура $\left(P_{0}^{\prime}, P_{1}^{\prime}, P_{2}^{\prime}\right)$ с полем $\tau^{\prime}$, удовлетворяющим условиям (2.2), и с оператором рекурсии $N$ таким, что $N P_{0}^{\prime}=P_{1}^{\prime}$ и $N P_{1}^{\prime}=\lambda P_{2}^{\prime}+\mu P_{0}^{\prime}$. Тогда тензоры $Q_{0}=P_{0}^{\prime}, Q_{1}=P_{1}^{\prime}$ и $Q_{2}=P_{2}^{\prime}+(\mu / \lambda) P_{0}^{\prime}$ таковы, что деформачионные соотношения (2.2) остаются справедливыми, и имеем $N Q_{0}=Q_{1}, N Q_{1}=\lambda Q_{2}$.

ДОКАЗАТЕЛЬСТВО состоит в прямом вычислении.

Полученные результаты свидетельствуют о том, что при заданной трехгамильтоновой структуре $\left(P_{0}, P_{1}, P_{2}\right)$ с деформационным полем $\tau$, удовлетворяюшим соотношениям (2.1), и в случае, когда деформация и трехгамильтонова структура сохраняются при погружении $\pi$, сушествует также и структура рекурсий, задаваемая оператором $N$ на редуцированном многообразии (возможно после масштабного преобразования). 


\section{3. НЕКОТОРЫЕ СВОЙСТВА КБГ-МОДЕЛИ}

КБГ-модель была введена в работах [4] и исследована более подробно в [5], [6].

Пусть даны два согласованных пуассоновых тензора $Q_{0}$ и $Q_{1}$. Векторное поле $X$ допускает формулировку в терминах КБГ-системы, если существуют такие три функции $\rho, H$ и $K$, что

$$
X=Q_{0} d H=\frac{1}{\rho} Q_{1} d K .
$$

Если многообразие $M$ четномерно, $\operatorname{dim} M=2 n$, то будем говорить, что формулировка в терминах КБГ-системы имеет максимальный ранг, если $Q_{0}$ и $Q_{1}$ невырожденны в каждой точке $m \in M$, а ассоциированньй с ними тензор $N=Q_{1} Q_{0}^{-1}$ (с исчезаюшим кручением Нийенхейса) имеет $n$ различных собственных значений $\lambda_{1}(m), \ldots, \lambda_{n}(m)$. Будем говорить, что формулировка в терминах КБГ-системы имеет пфаффианов вид, если $\rho=\prod_{i=1}^{n} \lambda_{i}$. Для КБГ-структуры максимального ранга можно ввести карту Дарбу-Нийенхейса $\left(\lambda_{i} ; \mu_{i}\right), i=1,2, \ldots, n$, такую, что $Q_{0}, Q_{1}$ и $N$ принимают в ней канонический вид:

$$
Q_{0}=\left(\begin{array}{cc}
0 & I \\
-I & 0
\end{array}\right), \quad Q_{1}=\left(\begin{array}{cc}
0 & \Lambda \\
-\Lambda & 0
\end{array}\right), \quad N=\left(\begin{array}{cc}
\Lambda & 0 \\
0 & \Lambda
\end{array}\right)
$$

где $I$ - единичная $(n \times n)$-матриша и $\Lambda=\operatorname{diag}\left(\lambda_{1}, \ldots, \lambda_{n}\right)[7]$.

ПреДЛОЖенИЕ 3.1 [5]. В переменных на карте Дарбу-Нийенхейса общее решение уравнения (3.1) в пфаффиановом случае задается функииями

$$
H=\sum_{i=1}^{n} \frac{f_{i}}{\Delta_{i}}, \quad K=\sum_{i=1}^{n} \frac{\rho}{\lambda_{i}} \frac{f_{i}}{\Delta_{i}},
$$

где $\Delta_{i}=\prod_{j \neq i}\left(\lambda_{i}-\lambda_{j}\right)$, а $f_{i}-$ произвольные функиии, зависящие не более чем от пары переменных $\left(\lambda_{i}, \mu_{i}\right)$. Более того, уравнения ГЯ допускают разделение переменных для обеих функций $H$ и $K$ в карте $(\lambda ; \mu)$.

Здесь и ниже будем называть функциями, имеюшими нормальный вид, функции вида (3.3) в данной карте. Из предложения 3.1 непосредственно вытекает

СлЕДСТвИЕ 3.1. Пусть $X=Q_{0} d H$ - гамильтоновы векторные поля, где величиньи $Q_{0}$ и $H$ задаются соответствующими формулами (3.2) и (3.3). Тогда существуют такие $Q_{1}$ и K, задаваемые соответствующими формулами (3.2) и (3.3), что $X=(1 / \rho) Q_{1} d K$.

Обратно, пусть $X=(1 / \rho) Q_{1} d K$ с $\rho=\prod_{i=1}^{n} \lambda_{i}$, а $Q_{1}$ и $K$ задаются соответствующими соотношениями (3.2) и (3.3). Тогда существуют величины $Q_{0}$ и $H$, задаваемые соответствующими формулами (3.2) и (3.3), такие, что $X=Q_{0} d H$.

В контексте приложения к ЛВ рассмотрим более подробно случай четырехмерного многообразия $(n=2)$. В этом случае возникают более обшие условия, приводяшие $\mathrm{K}$ тому, что гамильтоново векторное поле становится сепарабельным. 
ПРЕДЛОЖЕНИЕ 3.2. Пусть на четырехмерном многообразии задано гамильтоново векторное поле $X=Q_{0} d \widetilde{H}$, где $Q_{0}$ имеет канонический вид (3.2) в карте $(x ; y)$. Пусть гамильтониан $\widetilde{H}$ представляет собой линейную комбинацию двух функиий $\widehat{H}$ и $\widehat{K}$, приведенных к нормальной форме (3.3), т.е.

$$
\begin{aligned}
& \widetilde{H}(x ; y)=\alpha \widehat{H}(x ; y)+\beta \widehat{K}(x ; y), \quad \alpha, \beta=\text { const, } \\
& \widehat{H}(x ; y)=\frac{1}{x_{1}-x_{2}}\left(\hat{f}_{1}\left(x_{1}, y_{1}\right)-\hat{f}_{2}\left(x_{2}, y_{2}\right)\right), \\
& \widehat{K}(x ; y)=\frac{1}{x_{1}-x_{2}}\left(x_{2} \hat{f}_{1}\left(x_{1}, y_{1}\right)-x_{1} \hat{f}_{2}\left(x_{2}, y_{2}\right)\right) .
\end{aligned}
$$

Тогда отображсение $\Phi_{0}:(x ; y) \mapsto(\lambda ; \mu)$,

$$
\lambda_{i}=\frac{\beta}{\alpha+\beta x_{i}}, \quad \mu_{i}=-\frac{1}{\beta^{2}}\left(\alpha+\beta x_{i}\right)^{2} y_{i}, \quad i=1,2,
$$

симплектично для $Q_{0}$ (т.е. вид $Q_{0}$ сохраняется при действии $\left.\Phi_{0}\right)$, а гамильтониан $\widetilde{H}$ преобразуется под действием отображения $\Phi_{0}$ в функцию $H$, имеющую нормальный вид (3.3) с функииями

$$
f_{i}\left(\lambda_{i}, \mu_{i}\right)=-\beta \lambda_{i} \hat{f}_{i}\left(\frac{1}{\lambda_{i}}-\frac{\alpha}{\beta},-\lambda_{i}^{2} \mu_{i}\right), \quad i=1,2 .
$$

Тем самым векторное поле $X$ допускает формулировку в терминах КБГ-системь, а гамильтониан $\widetilde{H}$, как оказывается, допускает разделение переменных в карте $(\lambda ; \mu)$.

ДокАЗАТЕЛЬСТво. Прямыми вычислениями проверяется, что отображение $\Phi_{0}$ симплектично для $Q_{0}$ и что гамильтониан $\widetilde{H}$ принимает вид

$$
\widetilde{H}(x(\lambda ; \mu) ; y(\lambda ; \mu))=\frac{1}{\lambda_{1}-\lambda_{2}}\left(f_{1}\left(\lambda_{1}, \mu_{1}\right)-f_{2}\left(\lambda_{2}, \mu_{2}\right)\right)
$$

с функциями $f_{i}\left(\lambda_{i}, \mu_{i}\right)$, задаваемыми формулой (3.6). Согласно следствию 3.1 векторное поле $X=Q_{0} d H$ также допускает квазигамильтонову формулировку $X=(1 / \rho) Q_{1} d K$. Разделение переменных для $\widetilde{H}$ в карте $(\lambda ; \mu)$ следует при этом из предложения 3.1 .

СледСтвиЕ 3.2. Гамильтониан $\widetilde{H}$ также допускает разделение переменных в карте $(x ; y)$. Соответствующее уравнение ГЯ $\widetilde{H}(x ; \partial W / \partial x)=\tilde{h}$ имеет общее решение $W=W_{1}+W_{2}$, әде функиии $W_{1}$ и $W_{2}$ удовлетворяют разделяющим уравнениям Якоби [8]:

$$
\hat{f}_{i}\left(x_{i}, W_{i}^{\prime}\left(x_{i}\right)\right)=x_{i} \hat{h}-\hat{k}, \quad \alpha \hat{h}+\beta \hat{k}=\tilde{h}, \quad i=1,2 .
$$

В самом деле, отображение $\Phi_{0}$ представляет собой разделяющее отображение [9], т.е. оно отображает набор разделенных переменных в другой набор разделенных переменных. Поэтому с учетом вида (3.7) функции $\widetilde{H}$ можно легко проверить, что у уравнения ГЯ имеется полное решение $W\left(x_{1}, x_{2} ; \hat{h}, \hat{k}\right)=W_{1}\left(x_{1} ; \hat{h}, \hat{k}\right)+W_{2}\left(x_{2} ; \hat{h}, \hat{k}\right)$, где $\alpha \hat{h}+$ $\beta \hat{k}=\tilde{h}$, а функции $W_{1}$ и $W_{2}$ удовлетворяют разделяюшим уравнениям Якоби (3.8) для уравнений ГЯ $\widehat{H}(x ; \partial W / \partial x)=\hat{h}$ и $\widehat{K}(x ; \partial W / \partial x)=\hat{k}$. 
ПреДЛОЖениЕ 3.3. Зададим на четырехмерном многообразии поле $X=Q_{1} d \widetilde{H}$, представляющее собой гамильтоново векторное поле с матрицей $Q_{1}$ вида (3.2) в карте $(x ; y)$; пусть гамильтониан $\widetilde{H}$ задается при этом линейной комбинацией двух функиий $\widehat{H}$ и $\widehat{K}$ нормального вида (3.3), m.e.

$$
\begin{aligned}
\widetilde{H}(x ; y) & =\alpha \widehat{H}(x ; y)+\beta \widehat{K}(x ; y), \quad \alpha, \beta=\mathrm{const}, \\
\widehat{H}(x ; y) & =\frac{1}{x_{1}-x_{2}}\left(\hat{f}_{1}\left(x_{1}, y_{1}\right)-\hat{f}_{2}\left(x_{2}, y_{2}\right)\right), \\
\widehat{K}(x ; y) & =\frac{1}{x_{1}-x_{2}}\left(x_{2} \hat{f}_{1}\left(x_{1}, y_{1}\right)-x_{1} \hat{f}_{2}\left(x_{2}, y_{2}\right)\right) .
\end{aligned}
$$

Тогда отображение $\Phi_{1}:(x ; y) \mapsto(\lambda ; \mu)$, задаваемое соотношениями

$$
\lambda_{i}=\frac{1}{\alpha+\beta x_{i}}, \quad \mu_{i}=-\frac{1}{\beta}\left(\alpha+\beta x_{i}\right)^{2} \frac{y_{i}}{x_{i}}, \quad i=1,2,
$$

является отображсенем Дарбу для $Q_{1}$ (т.е. $Q_{1}$ отображается в матрииу $Q_{0}$ под действием $\left.\Phi_{1}\right)$, в то время как гамильтониан $\widetilde{H}$ переводится отображением $\Phi_{1}$ в функиию $Н$ нормального вида (3.3), в которой

$$
f_{i}\left(\lambda_{i}, \mu_{i}\right)=-\beta \lambda_{i} \hat{f}_{i}\left(\frac{1}{\beta}\left(\frac{1}{\lambda_{i}}-\alpha\right),-\left(\frac{1}{\lambda_{i}}-\alpha\right) \lambda_{i}^{2} \mu_{i}\right), \quad i=1,2 .
$$

Таким образом, векторное поле $X$ допускает формулировку в терминах КБГ-системь, а гамильтониан $\widetilde{H}$ допускает разделение переменных в карте $(\lambda ; \mu)$.

ДоКАЗАТЕЛЬСТВо. Можно проверить с помощью прямого вычисления, что отображение $\Phi_{1}$ задает преобразование Дарбу для $Q_{1}$ и гамильтониан $\widetilde{H}$ отображается при этом в функцию $H$ нормального вида с составляюшими $f_{i}$, заданными формулой $(3.10)$. Из следствия 3.1 и предложения 3.1 при этом следует, что гамильтониан $\widetilde{H}$ допускает разделение переменных в карте $(\lambda ; \mu)$.

ПРЕДЛОЖЕНИЕ 3.4. Пусть на четырехмерном многообразии поле $X=Q_{2} d \widetilde{G}$ представляет собой гамильтоново векторное поле с матрицей тензора $Q_{2}$ вида (3.2) в карте $(x ; y)$ и пусть

$$
\widetilde{G}=\alpha \widehat{K}+\beta \widehat{H}^{2},
$$

где $\widehat{H}$ и $\widehat{K}$ имеют нормальный вид (3.3). Тогда отображение $\Phi_{2}:(x ; y) \mapsto(\lambda ; \mu)$,

$$
\lambda_{i}=-\frac{1}{x_{i}}, \quad \mu_{i}=y_{i}, \quad i=1,2,
$$

является преобразованием Дарбу для $Q_{2}$ (т.е. оно отображает $Q_{2}$ в $\left.Q_{0}\right)$, а величина $\widetilde{G}$ отображается при преобразовании $\Phi_{2}$ в функиию

$$
G=\alpha H+\beta K^{2},
$$

где $Н$ и К имеют нормальный вид (3.3) и

$$
f_{i}\left(\lambda_{i}, \mu_{i}\right)=-\lambda_{i} \hat{f}_{i}\left(-\frac{1}{\lambda_{i}}, \mu_{i}\right), \quad i=1,2 .
$$

Функиия $\widetilde{G}$ обладает при этом свойством разделимости переменных в карте $(\lambda ; \mu)$. 
ДокАЗАТЕльСтво. Можно непосредственно проверить, что отображение $\Phi_{2}$ является преобразованием Дарбу для функции $Q_{2}$, а $\widetilde{G}$ при этом отображается в $G$. Рассмотрим уравнение ГЯ $G(\lambda ; \partial W / \partial \lambda)=g$ для гамильтониана $G$. Решение его примет вид $W=W_{1}+W_{2}$ с функциями $W_{1}$ и $W_{2}$, являющимися решениями уравнения с разделенными переменными

$$
f_{i}\left(\lambda_{i}, W_{i}^{\prime}\left(\lambda_{i}\right)\right)=\lambda_{i} h-k, \quad \alpha h+\beta k^{2}=g, \quad i=1,2 .
$$

\section{4. ТРЕХГАМИЛЬТОНОВА СТРУКТУРА ЛАГРАНЖЕВА ВОЛЧКА}

В системе, движущейся вместе с волчком, в которой оси координат направлены вдоль главных осей инерции волчка с фиксированной точкой $O$, ЛВ параметризуется парой $m=(\omega ; \gamma)$, где $\omega=\left(\omega_{1}, \omega_{2}, \omega_{3}\right)^{\mathrm{T}}$ и $\gamma=\left(\gamma_{1}, \gamma_{2}, \gamma_{3}\right)^{\mathrm{T}}$ суть соответственно угловая скорость и единичный вектор нормали. Обозначив через $\mu$ массу волчка, через $g$ ускорение свободного падения, через $J=\operatorname{diag}(A, A, c A)$ главную матрицу моментов инерции $(c \neq 1)$ и через $G=(0,0, a)^{\mathrm{T}}$ координату центра масс, выберем нормировку таким образом, чтобы удовлетворялось равенство $\mu a g=A$.

Уравнения Эйлера-Пуассона $d L_{O} / d t=M_{O}$ и $d \gamma / d t=0$ при этом принимают вид $d m / d t=X_{L}(m)$, где $X_{L}$ дается формулой

$$
X_{L}(m)=\left(-(c-1) \omega_{2} \omega_{3}-\gamma_{2},(c-1) \omega_{3} \omega_{1}+\gamma_{1}, 0 ; \gamma_{2} \omega_{3}-\gamma_{3} \omega_{2}, \gamma_{3} \omega_{1}-\gamma_{1} \omega_{3}, \gamma_{1} \omega_{2}-\gamma_{2} \omega_{1}\right)^{\mathrm{T}} \text {. }
$$

Векторное поле ЛВ $X_{L}$ допускает трехгамильтонову формулировку:

$$
X_{L}=P_{0} d h_{0}=P_{1} d h_{1}=P_{2} d h_{2} .
$$

В матрично-блочной форме записи согласованные пуассоновы тензоры имеют вид

$$
\begin{gathered}
P_{0}=\left(\begin{array}{ll}
0 & B \\
B & C
\end{array}\right), \quad P_{1}=\left(\begin{array}{cc}
-B & 0 \\
0 & \Gamma
\end{array}\right), \quad P_{2}=\left(\begin{array}{cc}
T & R \\
-R^{\mathrm{T}} & 0
\end{array}\right), \\
B=\left(\begin{array}{ccc}
0 & -1 & 0 \\
1 & 0 & 0 \\
0 & 0 & 0
\end{array}\right), \quad C=\left(\begin{array}{ccc}
0 & c \omega_{3} & -\omega_{2} \\
-c \omega_{3} & 0 & \omega_{1} \\
\omega_{2} & -\omega_{1} & 0
\end{array}\right), \quad \Gamma=\left(\begin{array}{ccc}
0 & \gamma_{3} & -\gamma_{2} \\
-\gamma_{3} & 0 & \gamma_{1} \\
\gamma_{2} & -\gamma_{1} & 0
\end{array}\right), \\
T=\left(\begin{array}{ccc}
0 & -c \omega_{3} & \omega_{2} / c \\
c \omega_{3} & 0 & -\omega_{1} / c \\
-\omega_{2} / c & \omega_{1} / c & 0
\end{array}\right), \quad R=\left(\begin{array}{ccc}
0 & -\gamma_{3} & \gamma_{2} \\
\gamma_{3} & 0 & -\gamma_{1} \\
-\gamma_{2} / c & \gamma_{1} / c & 0
\end{array}\right) .
\end{gathered}
$$

Соответствуюшие функции Гамильтона при этом имеют вид

$$
\begin{gathered}
h_{0}=\frac{1}{2} F_{4}+(c-1) F_{1} F_{3}, \quad h_{1}=\frac{1}{2} c(c-1) F_{1}^{3}-F_{3}-(c-1) F_{1} F_{2}, \quad h_{2}=F_{2}, \\
F_{1}=\omega_{3}, \quad F_{2}=\frac{1}{2}\left(\omega_{1}^{2}+\omega_{2}^{2}+c \omega_{3}^{2}\right)-\gamma_{3}, \\
F_{3}=\omega_{1} \gamma_{1}+\omega_{2} \gamma_{2}+c \omega_{3} \gamma_{3}, \quad F_{4}=\gamma_{1}^{2}+\gamma_{2}^{2}+\gamma_{3}^{2} .
\end{gathered}
$$

Функции $\left(F_{1}, F_{2}\right),\left(F_{1}, F_{4}\right)$ и $\left(F_{3}, F_{4}\right)$ суть функции Казимира для соответствующих структур $P_{0}, P_{1}$ и $P_{2}$. 
Гамильтонова формулировка для ЛВ по отношению к структуре $P_{2}$ является классической (см., например, [10]). Бигамильтонова формулировка по отношению к структурам $\left(P_{0}, P_{2}\right)$ была построена в работе [11] в полупрямом произведении $\mathfrak{s o}(3) \times \mathfrak{s o}(3)$ и в дальнейшем была переоткрыта в работе [12] в алгебро-геометрическом подходе. В работе [13] с помошью подходящей редукции пучка Ли-Пуассона, заданного в прямой сумме трех копий алгебр $\mathfrak{s o}(3)$, была построена трехгамильтонова формулировка по отношению к тензорам $\left(P_{0}, P_{1}, P_{2}\right)$.

Как показано в работе [13], трехгамильтонова структура ЛВ допускает деформацию вида $L_{\tau}\left(P_{2}\right)=2 P_{1}, L_{\tau}\left(P_{1}\right)=P_{0}, L_{\tau}\left(P_{0}\right)=0$, где $\tau$ задается в карте $(\omega ; \gamma)$ соотношением $\tau=\left(0,0,-2 / c ; \omega_{1}, \omega_{2}, c \omega_{3}\right)^{\mathrm{T}}$. Напротив, оператор рекурсии $N$, связываюший пуассоновы тензоры, не сушествует на многообразии $M$.

Пуассоновы пучки $P_{1}-\lambda P_{0}, P_{2}-\lambda P_{1}$ и $P_{2}-\lambda P_{0}$ суть три пуассоновых пучка типа Гельфанда-Захаревича; точнее, эти пучки лежат в классе полных систем Гельфанда-Захаревича без кручения, имеюших ранг 2 (см. [14]). Каждый из этих пучков допускает две полиномиальные функции Казимира, коэффициенты которых образуют цепочки Ленарда для каждого из пучков, которые строятся с помошью деформационного поля $\tau$. В графическом представлении цепочки Ленарда системы $\left(P_{0}, P_{1}\right)$ могут быть представлены в виде

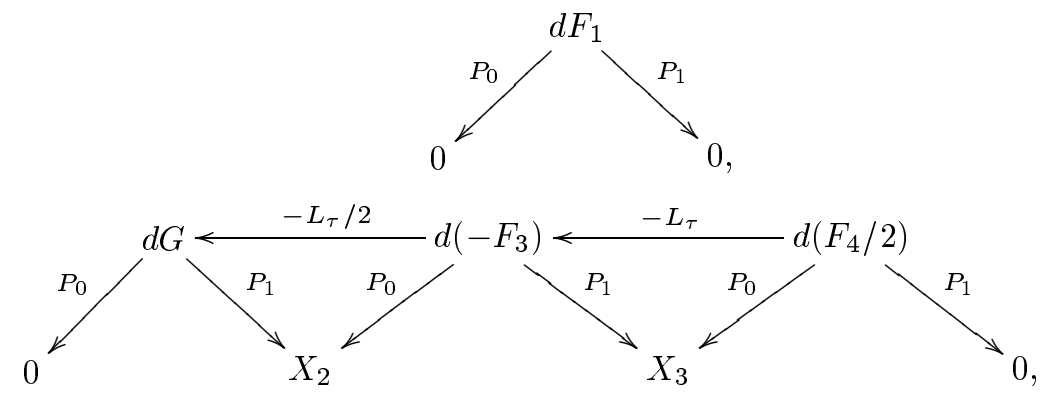

где $G=F_{2}+c(c-1) F_{1}^{2} / 2$. Цепочки Ленарда системы $\left(P_{1}, P_{2}\right)$ при этом имеют вид

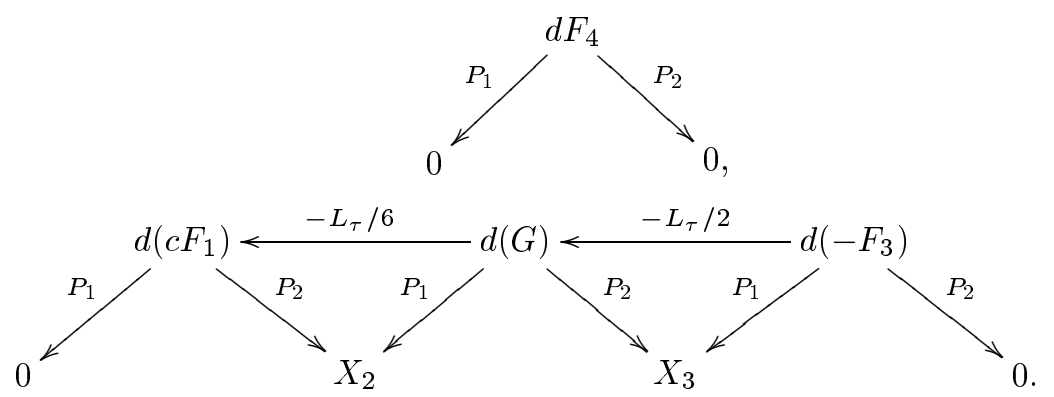

Таким образом, можно утверждать, что структуры $P_{1}-\lambda P_{0}$ и $P_{2}-\lambda P_{1}$ представляют собой пуассоновы пучки ранга 2 и типа $(1,5)$. Наконец, цепочки Ленарда структуры 
$\left(P_{0}, P_{2}\right)$ имеют вид

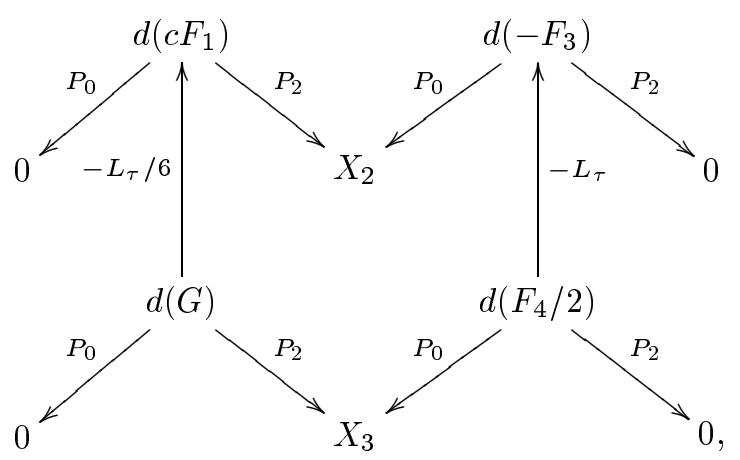

из которого следует, что структура $P_{2}-\lambda P_{0}$ представляет собой пуассонов пучок ранга 2 и типа $(3,3)$.

\section{5. РЕДУКЦИИ ТРЕХГАМИЛЬТОНОВОЙ И ДЕФОРМАЦИОННОЙ СТРУКТУР}

Трехгамильтонова структура $\left(P_{0}, P_{1}, P_{2}\right)$ ЛВ, равно как и деформационное поле $\tau$, допускает редукцию на четырехмерное многообразие $M^{\prime}$ (см. работу [1], где этот процесс редукции интерпретировался в терминах теоремы редукции Марсдена-Ратье).

Пусть $M^{\prime}$ представляет собой четырехмерное многообразие, параметризованное переменными карты $(x ; y)=\left(x_{1}, x_{2} ; y_{1}, y_{2}\right)$, а $\pi: M \mapsto M^{\prime}=M / \pi,(\omega ; \gamma) \mapsto(x ; y)$ задает сюръективное погружение, задаваемое формулами

$$
\begin{aligned}
& x_{1,2}=-\frac{1}{2}\left(c \omega_{3}-i \omega_{2}\right) \mp \frac{1}{2} \sqrt{\left(c \omega_{3}-i \omega_{2}\right)^{2}+4\left(\gamma_{3}-i \gamma_{2}\right)}, \\
& y_{1,2}=-\gamma_{1}-\frac{1}{2} \omega_{1}\left(c \omega_{3}-i \omega_{2}\right) \mp \frac{1}{2} \omega_{1} \sqrt{\left(c \omega_{3}-i \omega_{2}\right)^{2}+4\left(\gamma_{3}-i \gamma_{2}\right)} .
\end{aligned}
$$

Прямым вычислением можно получить следуюшее

ПРЕДЛОЖЕНИЕ 5.1. Пуассонов тензор $P_{0}$ и деформационное поле $\tau$ редуцируются под действием проекиии $\pi$ : проекиии тензорных полей при этом имеют вид

$$
P_{0}^{\prime}=-i\left(\begin{array}{cc}
0 & I \\
-I & 0
\end{array}\right), \quad \tau^{\prime}=(1,1,0,0)^{\mathrm{T}},
$$

где I - единичная $(2 \times 2)$-матрица. В силу леммы 2.2 структурь $P_{1}$ и $P_{2}$ также допускают проекиии: редуцированные тензорь $P_{1}^{\prime}$ и $P_{2}^{\prime}$ имеют вид

$$
P_{1}^{\prime}=-i\left(\begin{array}{cc}
0 & \mathcal{X} \\
-\mathcal{X} & 0
\end{array}\right), \quad P_{2}^{\prime}=-i\left(\begin{array}{cc}
0 & \mathcal{X}^{2} \\
-\mathcal{X}^{2} & 0
\end{array}\right),
$$

əде $\mathcal{X}=\operatorname{diag}\left(x_{1}, x_{2}\right)$.

Более того, проекция $\pi$ сохраняет соотношения деформации. Поскольку структура $P_{0}^{\prime}$, очевидно, имеет нулевое ядро, тензор $N=P_{1}^{\prime} P_{0}^{\prime-1}$ существует и имеет нулевое кручение; при этом легко проверить, что $N P_{0}^{\prime}=P_{1}^{\prime}$ и $N P_{1}^{\prime}=P_{2}^{\prime}$. Поэтому данная структура представляет собой частный случай структуры, обсуждавшейся в лемме 2.3 , с параметрами $a=2$ и $\beta=0$. 


\section{6. РЕДУКЦИИ ВЕКТОРНОГО ПОЛЯ $X_{L}$ НА СИМПЛЕКТИЧЕСКИЕ ЛИСТЫ}

Рассмотрим в данном разделе редукцию системы ЛВ на симплектические листы $S_{i}$ пуассоновых тензоров $P_{i}, i=0,1,2$. Каждый лист $S_{i}$ представляет собой четырехмерное подмногообразие многообразия $M$, задаваемое набором уровней двух функций Казимира структуры $P_{i}$. В силу формул (4.1) симплектические листы задаются соотношениями

$$
\begin{aligned}
& S_{0}=\left\{m \in M \mid \omega_{3}=C_{1}, \omega_{1}^{2}+\omega_{2}^{2}+c \omega_{3}^{2}-2 \gamma_{3}=2 C_{2}\right\}, \\
& S_{1}=\left\{m \in M \mid \omega_{3}=C_{1}, \gamma_{1}^{2}+\gamma_{2}^{2}+\gamma_{3}^{2}=C_{4}\right\}, \\
& S_{2}=\left\{m \in M \mid \omega_{1} \gamma_{1}+\omega_{2} \gamma_{2}+c \omega_{3} \gamma_{3}=C_{3}, \gamma_{1}^{2}+\gamma_{2}^{2}+\gamma_{3}^{2}=C_{4}\right\},
\end{aligned}
$$

в которых $C_{1}, C_{2}, C_{3}$ и $C_{4}$ суть фиксированные значения соответствуюших функций Казимира $F_{1}, F_{2}, F_{3}$ и $F_{4}$. Поскольку эти интегралы движения находятся в инволюции по отношению к каждой из структур $P_{i}$, набор уровней, образуемых остающимися двумя интегралами движения на листе $S_{i}$, представляет собой лист $\Lambda_{i}$ лагранжева слоения листа $S_{i}$. Заметим, что для всякой точки $m \in M$ лагранжевы листы $\Lambda_{0}, \Lambda_{1}$ и $\Lambda_{2}$, проходящие через $m$, совпадают. Более того, с учетом теоремы редукции Марсдена-Ратье, можно доказать следующий результат.

ПРЕДЛОЖЕНИЕ 6.1 [5]. Симплектические листь $S_{0}, S_{1}$ u $S_{2}$ (локально) диффеоморфны четырехмерному многообразию $M^{\prime}=M / \pi$.

Обратившись к явной конструкции, получим, что если $\left(x_{1}, x_{2} ; y_{1}, y_{2}\right)$ задает карту на многообразии $M^{\prime}$, то можно проверить, что симплектические листы допускают следующие параметризации.

Лемма 6.1. Симплектический лист $S_{0}$ параметризуется отображением $\Psi_{0}$ : $M^{\prime} \mapsto M,(x ; y) \mapsto(\omega ; \gamma)$, задаваемым формулами

$$
\begin{array}{ccc}
\omega_{1}=\frac{y_{2}-y_{1}}{x_{2}-x_{1}}, & \omega_{2}=-i\left(x_{1}+x_{2}+c C_{1}\right), & \omega_{3}=C_{1}, \\
\gamma_{1}=\frac{x_{1} y_{2}-x_{2} y_{1}}{x_{2}-x_{1}}, & \gamma_{2}=\frac{i}{2}\left[x_{1}^{2}+x_{2}^{2}+g_{1}(x ; y)\right], & \gamma_{3}=-\frac{1}{2}\left[\left(x_{1}+x_{2}\right)^{2}+g_{1}(x ; y)\right], \\
g_{1}(x ; y)=-\frac{\left(y_{2}-y_{1}\right)^{2}}{\left(x_{2}-x_{1}\right)^{2}}+2 c C_{1}\left(x_{1}+x_{2}\right)+2 C_{2}+c(c-1) C_{1}^{2} .
\end{array}
$$

Симплектический лист $S_{1}$ параметризуется отображсением $\Psi_{1}$, задаваемым формулами

$$
\begin{aligned}
& \omega_{1}=\frac{y_{2}-y_{1}}{x_{2}-x_{1}}, \quad \omega_{2}=-i\left(x_{1}+x_{2}+c C_{1}\right), \quad \omega_{3}=C_{1}, \\
& \gamma_{1}=\frac{x_{1} y_{2}-x_{2} y_{1}}{x_{2}-x_{1}}, \quad \gamma_{2}=-\frac{i}{2}\left[x_{1} x_{2}-g_{2}(x ; y)\right], \quad \gamma_{3}=-\frac{1}{2}\left[x_{1} x_{2}+g_{2}(x ; y)\right], \\
& g_{2}(x ; y)=-\frac{\left(x_{2} y_{1}-x_{1} y_{2}\right)^{2}}{x_{1} x_{2}\left(x_{2}-x_{1}\right)^{2}}+\frac{C_{4}}{x_{1} x_{2}} \text {. }
\end{aligned}
$$


Симплектический лист $S_{2}$ параметризуется отображением $\Psi_{2}$, задаваемым формулами

$$
\begin{aligned}
& \omega_{1}=\frac{y_{2}-y_{1}}{x_{2}-x_{1}}, \quad \omega_{2}=-\frac{i}{2}\left[x_{1}+x_{2}+g_{3}(x ; y)\right], \quad \omega_{3}=-\frac{1}{2 c}\left[x_{1}+x_{2}-g_{3}(x ; y)\right], \\
& \gamma_{1}=\frac{x_{1} y_{2}-x_{2} y_{1}}{x_{2}-x_{1}}, \quad \gamma_{2}=-\frac{i}{2}\left[x_{1} x_{2}-g_{2}(x ; y)\right], \quad \gamma_{3}=-\frac{1}{2}\left[x_{1} x_{2}+g_{2}(x ; y)\right], \\
& g_{3}(x ; y)=\frac{x_{1}^{2} y_{2}^{2}-x_{2}^{2} y_{1}^{2}}{x_{1}^{2} x_{2}^{2}\left(x_{2}-x_{1}\right)}-\frac{2 C_{3}}{x_{1} x_{2}}+\frac{x_{1}+x_{2}}{x_{1}^{2} x_{2}^{2}} C_{4} .
\end{aligned}
$$

Используя эти параметризации, можно показать, что ЛВ допускает формулировку в терминах КБГ-системы на каждом из симплектических листов. Тогда следуюшие три предложения легко доказываются прямыми вычислениями.

ПРЕДЛОЖениЕ 6.2. Векторное поле $X_{L}$, будучи ограничено на $S_{0}$, имеет вид

$$
X_{L}=P_{0}^{\prime} d \widetilde{H}
$$

əде структура $P_{0}^{\prime}$ задается формулой (5.1). Гамильтониан $\widetilde{H}=\left.h_{0}\right|_{S_{0}}$ имеет вид

$$
\widetilde{H}(x ; y)=(c-1) C_{1} \widehat{H}(x ; y)+\widehat{K}(x ; y),
$$

əде

$$
\begin{aligned}
\widehat{H}(x ; y) & =\left.F_{3}\right|_{S_{0}}=\frac{1}{x_{1}-x_{2}}\left(\hat{f}\left(x_{1}, y_{1}\right)-\hat{f}\left(x_{2}, y_{2}\right)\right), \\
\widehat{K}(x ; y) & =\left.\frac{F_{4}}{2}\right|_{S_{0}}=\frac{1}{x_{1}-x_{2}}\left(x_{2} \hat{f}\left(x_{1}, y_{1}\right)-x_{1} \hat{f}\left(x_{2}, y_{2}\right)\right), \\
\hat{f}(\xi, \eta) & =-\frac{1}{2} \eta^{2}+\frac{1}{2} \xi^{4}+c C_{1} \xi^{3}+\left(C_{2}+\frac{1}{2} c(c-1) C_{1}^{2}\right) \xi^{2} .
\end{aligned}
$$

Тем самым имеет место частный случай ситуации из предложения 3.2 при $\alpha=$ $(c-1) C_{1}$ и $\beta=1$. Отсюда можно заключить, что поле $\left.X_{L}\right|_{S_{0}}$ в карте $(\lambda ; \mu)$, задаваемой формулами (3.5), представляет собой разделяющееся КБГ-векторное поле.

ЗАмЕчАниЕ. Векторное поле $\left.X_{L}\right|_{S_{0}}$ также допускает разделение переменных в карте $(x ; y)$. В самом деле, как следует из уравнений $(3.8)$ и выражения $(6.3)$ для $\hat{f}$, решение $W$ уравнения ГЯ для гамильтониана $\widetilde{H}$ имеет вид $W=W_{1}+W_{2}$, где функции $W_{1}$ и $W_{2}$ могут быть вычислены в квадратурах при решении системы обыкновенных дифференциальных уравнений, получаемой при замене величин $y_{i}$ на величины $\partial W / \partial x_{i}$ в уравнении

$$
y_{i}^{2}=x_{i}^{4}+2 c C_{1} x_{i}^{3}+\left[2 C_{2}+c(c-1) C_{1}^{2}\right] x_{i}^{2}-2 \hat{h} x_{i}+2 \hat{k}, \quad i=1,2 .
$$

Здесь $\tilde{h}=(c-1) C_{1} \hat{h}+\hat{k}$, а $\tilde{h}, \hat{h}$ и $\hat{k}$ суть соответствующие значения гамильтонианов $\widetilde{H}$, $\widehat{H}$ и $\widehat{K}$, ограниченных на лагранжев лист $\Lambda_{0}\left(C_{3}, C_{4}\right)$. Можно теперь установить связь с методом разделения переменных Склянина [8]. В самом деле, сравнивая (6.4) со спектральной кривой, получаемой из пары Лакса [11], можно непосредственно заключить, что переменные разделения $(x, y)$ удовлетворяют уравнению спектральной кривой, ограниченной на лист $\Lambda_{0}\left(C_{3}, C_{4}\right)$.

Рассмотрим теперь редукцию на симплектический лист $S_{1}$. 
ПРЕДЛОЖенИЕ 6.3. Векторное поле $X_{L}$, ограниченное на лист $S_{1}$, имеет вид

$$
X_{L}=P_{1}^{\prime} d \widetilde{H}
$$

где $P_{1}^{\prime}$ задается формулой (5.2). Гамильтониан $\widetilde{H}=\left.h_{1}\right|_{S_{1}}$ может быть представлен в виде

$$
\begin{aligned}
\widetilde{H}(x ; y) & =(c-1) C_{1} \widehat{H}(x ; y)+\widehat{K}(x ; y), \\
\widehat{H}(x ; y) & =\frac{1}{x_{1}-x_{2}}\left(\hat{f}\left(x_{1}, y_{1}\right)-\hat{f}\left(x_{2}, y_{2}\right)\right), \\
\widehat{K}(x ; y) & =\frac{1}{x_{1}-x_{2}}\left(x_{2} \hat{f}\left(x_{1}, y_{1}\right)-x_{1} \hat{f}\left(x_{2}, y_{2}\right)\right), \\
\hat{f}(\xi, \eta) & =-\frac{1}{2 \xi} \eta^{2}+\frac{1}{2} \xi^{3}+c C_{1} \xi^{2}+\frac{C_{4}}{2 \xi}-\frac{1}{2} c(c-1)(c-2) C_{1}^{3} .
\end{aligned}
$$

При этом возникает частный случай ситуации из предложения $3.3 \mathrm{c}$ параметрами $\alpha=(c-1) C_{1}$ и $\beta=1$; поэтому можно прийти к заключению, что карта $(\lambda ; \mu)$, определенная формулами $(3.9)$, задает разделение переменных для гамильтониана $\widetilde{H}$. Решение уравнения ГЯ для $\widetilde{H}$ задается формулами:

$$
\begin{gathered}
W\left(\lambda_{1}, \lambda_{2} ; \tilde{h}, \hat{k}\right)=\int^{\lambda_{1}} \sqrt{\hat{\varphi}_{1}(\xi)} d \xi+\int^{\lambda_{2}} \sqrt{\hat{\varphi}_{1}(\xi)} d \xi \\
\hat{\varphi}_{1}(\xi)=\frac{1}{\xi^{4}}\left(\left(\frac{1}{\xi}-\alpha\right)^{2}+2 c C_{1}\left(\frac{1}{\xi}-\alpha\right)-\frac{\alpha c(c-2) C_{1}^{2}-2 \tilde{h}+2 \hat{k} / \xi}{1 / \xi-\alpha}+\frac{C_{4}}{(1 / \xi-\alpha)^{2}}\right),
\end{gathered}
$$

где $\tilde{h}$ и $\hat{k}$ - значения гамильтонианов $\widetilde{H}$ и $\widehat{K}$ на лагранжевом листе $\Lambda_{1}\left(C_{2}, C_{3}\right)$.

Наконец, обратившись к редукции на симплектический лист $S_{2}$, получим следуюший результат.

ПреДЛОЖенИЕ 6.4. Векторное поле $X_{L}$, ограниченное на лист $S_{2}$, принимает вид

$$
X_{L}=P_{2}^{\prime} d \widetilde{G}
$$

где $P_{2}^{\prime}$ задается формулой (5.2). Гамильтониан $\widetilde{G}=\left.h_{2}\right|_{S_{2}}$ может быть представлен в виде

$$
\begin{aligned}
\widetilde{G} & =-\frac{1}{2 c}(c-1) \widehat{H}^{2}+\widehat{K} \\
\widehat{H}(x ; y) & =-\left.c F_{1}\right|_{S_{2}}=\frac{1}{x_{1}-x_{2}}\left(\hat{f}\left(x_{1}, y_{1}\right)-\hat{f}\left(x_{2}, y_{2}\right)\right), \\
\widehat{K}(x ; y) & =\left.G\right|_{S_{2}}=\frac{1}{x_{1}-x_{2}}\left(x_{2} \hat{f}\left(x_{1}, y_{1}\right)-x_{1} \hat{f}\left(x_{2}, y_{2}\right)\right), \\
\hat{f}(\xi, \eta) & =-\frac{1}{2}\left(\frac{\eta^{2}}{\xi^{2}}-\xi^{2}+2 \frac{C_{3}}{\xi}-\frac{C_{4}}{\xi^{2}}\right) .
\end{aligned}
$$


При этом получается в точности частньй случай ситуации из предложения 3.4 при подстановке $\beta=-(c-1) / 2 c$ и $\alpha=1$; также в этом случае векторное поле $\left.X_{L}\right|_{S_{2}}$ оказывается разделимым в карте $(\lambda ; \mu)$, задаваемой формулами (3.11). Решение уравнения ГЯ для гамильтониана $\widehat{K}$ при этом имеет вид

$$
\begin{gathered}
W\left(\lambda_{1}, \lambda_{2} ; h, k\right)=\int^{\lambda_{1}} \sqrt{\hat{\varphi}_{2}(\xi)} d \xi+\int^{\lambda_{2}} \sqrt{\hat{\varphi}_{2}(\xi)} d \xi \\
\hat{\varphi}_{2}(\xi)=C_{4}+\frac{2 C_{3}}{\xi}+\frac{2 \hat{k}}{\xi^{2}}-\frac{2 \hat{h}}{\xi^{3}}+\frac{1}{\xi^{4}}
\end{gathered}
$$

где $\hat{h}$ и $\hat{k}$ суть значения соответствующих гамильтонианов $\widehat{H}$ и $\widehat{K}$ на лагранжевом листе $\Lambda_{2}\left(C_{1}, C_{2}\right)$.

ЗАмЕчАнИЕ. В отличие от случая структуры $S_{0}$, для структур $S_{1}$ и $S_{2}$ мы получили, что векторное поле ЛВ $X_{L}$ оказывается разделимым в карте $(\lambda ; \mu)$, но не допускает разделения переменных в карте $(x ; y)$, в которой оно не допускает гамильтоновой формулировки относительно канонического пуассонова тензора $Q_{0}$.

Благодарности. Работа была частично поддержана M.I.U.R. (исследовательский проект "Геометрия интегрируемых систем") и грантом G.N.F.M. I.N.D.A.M.

\section{Список литературы}

[1] C. Morosi, G. Tondo. J. Phys. A. 2002. V. 35. P. 1741-1750.

[2] G. Tondo. J. Phys. A. 1995. V. 28. P. 5097-5115; Г. Фальки, Ф. Магри, Г. Тондо. ТМФ. 2000. T. 122. № 2. C. 212-230; G. Falqui, F. Magri, M. Pedroni, G. Zubelli. Regul. Chaotic Dyn. 2000. V. 5. P. 33-52.

[3] F. Magri, C. Morosi. A geometrical characterization of integrable Hamiltonian systems through the theory of Poisson-Nijenhuis manifolds. Quaderno 19/S, Dip. Mat. Milano: Università di Milano, 1984.

[4] R. Caboz, V. Ravoson, L. Gavrilov. J. Phys. A. 1991. V. 24. P. L523-L525; R. Brouzet, R. Caboz, J. Rabenivo, V. Ravoson. J. Phys. A. 1996. V. 29. P. 2069-2075.

[5] C. Morosi, G. Tondo. J. Phys. A. 1997. V. 30. P. 2799-2806.

[6] G. Tondo, C. Morosi. Rep. Math. Phys. 1999. V. 44. P. 255-266.

[7] F. Magri, T. Marsico. Some developments of the concept of Poisson manifolds in the sense of A. Lichnerowitz. In: Gravitation, Electromagnetism, and Geometric Structures. Ed. G. Ferrarese. Bologna: Pitagora, 1996. P. 207-222.

[8] E. K. Sklyanin. Progr. Theor. Phys. Suppl. 1995. V. 118. P. 35-60.

[9] S. Benenti. J. Math. Phys. 1997. V. 38. P. 6578-6602.

[10] L. Gavrilov, A. Zhivkov. Enseign. Math. 1998. V. 44. P. 133-170.

[11] T. Ratiu. Am. J. Math. 1982. V. 104. P. 409-448.

[12] C. Médan. Phys. Lett. A. 1996. V. 215. P. 176-180.

[13] G. Magnano. Acc. Sc. Torino-Mem. Sc. Fis. 1995, 1996. V. 19, 20. P. 159-209.

[14] I. M. Gel'fand, I. S. Zakharevich. Selecta Math. (N. S.). 2000. V. 6. P. 131-183. 